\title{
Existence Theorem for Abstract Measure Delay Integro-Differential Equations
}

\author{
S. S. Bellale ${ }^{1}$, S. B. Birajdar ${ }^{2}$, D. S. Palimkar ${ }^{3}$ \\ ${ }^{1}$ Mathematics Research Centre, Dayanand Science College, Maharashtra, India \\ ${ }^{2}$ Department of Mathematics, Bidve Engineering College, Latur, Maharashtra, India \\ ${ }^{3}$ Department of Mathematics, Vasantrao Naik College, Nanded, Maharashtra, India \\ Email address: \\ sidhesh.bellale@gmail.com (S. S. Bellale),1sbidave@gmail.com (S. B. Birajdar),dspalimkar@rediffmail.com (D. S. Palimkar)
}

\section{To cite this article:}

S. S. Bellale, S. B. Birajdar, D. S. Palimkar. Existence Theorem for Abstract Measure Delay Integro-Differential Equations. Applied and Computational Mathematics. Vol. 4, No. 4, 2015, pp. 225-231. doi: 10.11648/j.acm.20150404.11

\begin{abstract}
In this paper, we have proved the existence and uniqueness results for an abstract measure delay integro-differential equation by using Leray-Schauder nonlinear alternative under certain Caratheodory conditions. The various aspects of the solutions of the abstract measure integro-differential equations have been studied in the literature using the various fixed point techniques such as Schauder,s fixed point principle and Banach contraction mapping principal etc. In this paper we have proved existence and uniqueness condition for Abstract Measure delay integro-differential equations.
\end{abstract}

Keywords: Time Scale, Abstract Measure Integro-Differential Equation, Abstract Measure Delay Integro-Differential Equation, Existence Theorem and Extermal Solutions

\section{Introduction}

The concept of stability has been widely used by many scientists under various model formulations. Absolute stability was originally formulated by Lur'e and Postnikov and is connected between with engineering and mathematical considerations. From a mathematical point of view, one arrives at this concept from of continuity. In our study we shall be concerned with a view of such physical phenomena.

Functional integro-differential equations with delay is a hereditary system in which the rate of charge or the derivative of the unknown function or set function depends upon the past history. The functional integro-differential equations of neutral type is a hereditary system in which the derivative of the unknown function is determined by the values of a state variable as well as the derivative of the state variable over some past interval in the phase space. Although the general theory and the basic result for integro-differential equations have now been thoroughly investigated, the study of functional integro-differential equations has not been complete yet. In recent years, this has been an increasing interest for such equations among the mathematicians all over the word.

The study of abstract measure integro-differential equations is initiated and developed at length in a series of papers by
Dhage $[1,2,3]$. The study of abstract measure delay differential equations was initiated by Joshi $[9,10]$, Shendge and Joshi [14] and Bellale $[4,5,6]$.

Using the approach of above mentioned papers, in this paper, we prove the existence and stability results for a abstract measure delay integro-differential equations.

\section{Preliminaries}

Let $R$ denote the real line, $R^{n}$ an Euclidean space with respect to the norm $|\cdot|_{n}$ defined by

$$
|x|_{n}=\max \left\{\left|x_{1}\right|, \ldots \ldots \ldots,\left|x_{n}\right|\right\}
$$

For $x=\left(x, \ldots \ldots \ldots, x_{n}\right) \in R^{n}$.

Let $\mathrm{X}$ be a real Banach space with any convenient norm $\|\cdot\|$. For any two points $x, y$ in $\mathrm{X}$, the segments $\overline{x y}$ in $\mathrm{X}$ is defined by

$$
\overline{x y}=\{z \in X \mid z=x+r(y-x), 0 \leq r \leq 1\} .
$$

Let $x_{0}$ and $y_{0}$ be two fixed points in $\mathrm{X}$, such that $\overline{0 y_{0}} \subset \overline{0 x_{0}}$, where 0 is the zero vector of $\mathrm{X}$. Let $\mathrm{z}$ be a point of 
$\mathrm{X}$, such that $\overline{0 x_{0}} \subset \overline{0 z}$. For this $\mathrm{z}$ and $x \in \overline{y_{0} z}$, define the sets $S_{x}$ and $\bar{S}_{x}$ as follows.

$$
\begin{aligned}
& S_{x}=\{r x:-\infty<r<1\} \\
& \bar{S}_{x}=\{r x:-\infty<r \leq 1\}
\end{aligned}
$$

For $x_{1}, x_{2} \in \overline{y_{0} z}$, we write $x_{1}<x_{2}\left(\operatorname{or} x_{2}>x_{1}\right)$ if $\overline{y_{0} x_{1}} \subset \overline{y_{0} x_{2}}$. Let the positive number $\left\|x_{0}-y_{0}\right\|$ be denoted by $\omega$. For each $x \in \overline{x_{0} z}, z>x_{0}$, let $x_{\omega}$ denote that element of $\overline{y_{0} z}$ which

$$
x_{\omega}<x,\left\|x-x_{\omega}\right\|=\omega
$$

Note that, $x_{\omega}$ and $\omega x$ are not the same points unless $\omega=0$ and $x=0$.

Let $\mathrm{M}$ denote the $\sigma$-algebra of all subsets of $\mathrm{X}$ so that (X, M) becomes a measurable space. Let $c a(X, M)$ by

$$
\|p\|=|p|_{n}(X)
$$

Where $|p|$ is a total variation measure of $\mathrm{p}$ and is given by

$$
|p|_{n}(X)=\sum_{i=1}^{\infty}\left|p\left(E_{i}\right)\right|_{n}
$$

For all $E_{i} \subset X$ with $X=\bigcup_{i=1}^{\infty} E_{i}, E_{i} \cap E_{i}=\theta$ for $i \neq j$. It is known that $c a(X, M)$ is a Banach space with respect to the norm $\|\cdot\|$ defined by (2.2). Let $\mu$ be a $\sigma$ finite measure on $\mathrm{X}$ and let $p \in c a(X, M)$. We say $\mathrm{p}$ is absolutely continuous with respect to the measure $\mu$ if $\mu(E)=0$ implies $p(E)=0$ for all $E \in M$. In this case, we write $p<<\mu$.

For a fixed $x_{0} \in X$, let $M_{0}$ be the smallest $\sigma$-algebra on $\bar{S}_{x 0}$, containing $\left\{x_{0}\right\}$ and the sets $S_{x}, x \in \overline{y_{0} x_{0}}$. Let $z \in X$ be such that $z>x_{0}$ and let $M_{z}$ denote the $\sigma$-algebra of all sets containing $M_{0}$ and the sets of the form $\bar{S}_{x}$ for $x \in \overline{x_{0} z}$. We define the set $B_{H}$ and $C_{H}$ by

$$
B_{H}=\{\mu \in R \| \mu \mid<H\},
$$

$$
B_{H}=\left\{p \in c a\left(\bar{S}_{z}, M_{z}\right) \mid\|q\|+C<H\right\}
$$

Where $H>0$ is given (large enough) and $C>0$.

Finally let, $L_{\mu}^{1}\left(S_{z}, R\right)$ denote the space of all $\mu$ integrable nonnegative real valued functions $\mathrm{h}$ on $S_{z}$ with the norm $\|\cdot\|_{L_{\mu}^{1}}$, defined by

$$
\|h\|_{L_{\mu}^{1}}=\int_{S_{z}}|h(x)| d \mu
$$

\section{Statement of the Problem}

Let $\mu$ be a $\sigma$ finite real measure on $\mathrm{X}$. Given a $p \in A C^{\prime}(1(X, M))$ with $p<<\mu$ consider the following abstract measure delay integro differentiate equation involving the delay $\omega$,

$$
\frac{d p}{d \mu}=\int_{\bar{S}_{\omega}} f\left(t, p\left(\bar{S}_{t}\right), \int_{\bar{S}_{x_{\omega}}} k\left(t, p\left(\bar{S}_{t_{\omega}}\right) d \mu\right) d \mu\right.
$$

a.e. $\{\mu\}$ on $\overline{x_{o} z}$

$$
p(E)=q(E) \quad E \in M_{0},
$$

Where $\mathrm{q}$ is a given known vector measure, $\frac{d p}{d \mu}$ is RadonNikodym derivative of $\mathrm{p}$ with respect to $\mu$ and the function $F: S_{z} \times R \times R \rightarrow R \quad$ is such that $x \rightarrow f\left(t, p\left(\bar{S}_{t}\right), \int_{\bar{S}_{t_{\omega}}} k\left(t, p\left(\bar{S}_{t_{\omega}}\right)\right) d \mu\right)$ is $\mu$ integrable for each $p \in A C^{\prime}\left(S_{z}, M_{z}\right)$.

Definition 3.1 :-

Given an initial real measure $\mathrm{q}$ on $M_{0}$, a vector $p \in A C^{\prime}\left(S_{z}, M_{z}\right)(z>x)$ is said to be a solution of delay (1.1) if

$$
p(E)=q(E), E \in M_{0}
$$

$p<<\mu$ on $\overline{x_{0} z}$

Remark 3.1 :- The delay integro-Differential equation (3.1) is equivalent to the abstract measure delay integro-differential equation.

and

$$
p(E)=\left\{\int _ { E } \left(\int_{S x_{\omega}} f\left(t, p\left(\bar{S}_{t}\right), \int_{\bar{S}_{t_{\omega}}} k\left(t, p\left(\bar{S}_{t_{\omega}}\right)\right) d \mu\right) d \mu ; E \in M_{z}, E \subset \overline{x_{0} z}=\left\{q(E) \quad ; E \in M_{0}\right.\right.\right.
$$

A solution $\mathrm{p}$ of delay integro-differential equation (3.1) on $\overline{x_{0} z}$ will be denoted by $p\left(\bar{S} x_{0}, q\right)$

We apply the Schauder's fixed point theorem foe formulating the main existence result for the delay integro-differential equation (3.1). Before stating this result, we recall definition.

Definition 3.2. :-

An operator $Q$ on a Banach space $X$ into it self is called compact if for any bounded subset of $\mathrm{S}$ of $\mathrm{X}$. Q (X) is a 
relatively compact subset of X. Q is called totally bounded if $\mathrm{Q}(\mathrm{S})$ is a totally bounded subset of $\mathrm{X}$ for each bounded subset $\mathrm{S}$ of $\mathrm{X}$. If $\mathrm{Q}$ is continuous and totally bounded, then it is called completely continuous on $\mathrm{X}$.

Every compact operator is totally bounded, but the converse may not be true however both notions coincide on bounded subset of $X$.

Theorem 3.1 :-

Let $\mathrm{S}$ be a non-empty, closed convex and bounded subset of the Banach space $\mathrm{X}$ and let $Q: S \rightarrow S$ be a continuous and compact operator. Then the operator equation $Q x=x$ has a solutions.

Now we shall prove the main existence theorem for the delay (3.1) under suitable conditions of the function R.

\section{Existence and Uniqueness Theorem}

Definition 4.1:-

A function $\beta: S_{z} \times R^{n} \times R^{n} \rightarrow R^{n}$ is said to satisfy conditions of Caratheodory or simply it is Caratheodory if $x \rightarrow \beta(x, y, z)$ is $\mu$ measurable for each $(y, z) \in R^{n} \times R^{n}$, and $(y, z) \rightarrow \beta(x, y, z)$ is continuous for almost everywhere $\mu$ on $x \in \overline{x_{0} z}$.

Definition 4.2:-

A Caratheodory function $\mathrm{f}$ is called $L_{\mu}^{1}$ Caratheodory if

For each given real number $p>0$ there exists a function $h_{p} \in L_{\mu}^{1}\left(S_{z}, R\right)$ such that

$$
|\beta(x, y, z)|_{n} \leq h_{p}(x) \text { a.e. }[\mu] x \in \overline{x_{0} z},
$$

for all $y, z \in R$ with $|y|_{n} \leq p,|z|_{n} \leq p$.

Definition 4.3 :-

A function $\beta: S_{z} \times R^{n} \times R^{n} \rightarrow R^{n} \quad$ is called $L_{\mu}^{1}\left(R^{n}\right)$ Caratheodory if there exists a function $h \in L_{\mu}^{1}\left(S_{z}, R\right)$ such that.

$$
|\beta(x, y, z)|_{n} \leq h(x) \text { a.e. }[\mu] x \in \overline{x_{0} z}
$$

for all $y, z \in R^{n}$

Consider the following set of assumptions.

$$
\left(A_{0}\right) \mu\left(\left\{x_{0}\right\}\right)=0,\left(A_{1}\right) \text { For any } \delta>x_{0},
$$

the $\sigma$-algebra $\mathrm{M}_{\mathrm{z}}$ is compact with respect to topology generated by the pseudo-metric defined by

$$
d\left(E_{1} E_{2}\right)=|\mu|_{n}\left(E_{1} \Delta E_{2}\right), \quad E_{1}, E_{2} \in M_{z}
$$

$\left(A_{2}\right) \quad q$ is continuous on $M_{z}$ with respect to the pseudo-metric d defined in $\left(B_{1}\right)$

$\left(A_{3}\right)$ The function $f(x, y, z)$ is $L_{\mu}^{1}\left(R^{n}\right)$-Caratheodory.

$\left(A_{4}\right)$ The function $f$ is continuous and there exist functions $l_{1}, l_{2} \in L_{\mu}^{1}\left(S_{z} R^{+}\right)$such

$$
\begin{aligned}
& \left|f(x, y, z)-f\left(x, y_{2}, z_{2}\right)\right|_{n} \leq l_{1}(x)\left|y_{1}-y_{2}\right|_{n}+l_{2}(x)\left|z_{1}-z_{2}\right|_{n} \\
& \quad \text { for all } y_{1}, z_{1} y_{2} z_{2} \in R^{n}
\end{aligned}
$$

Theorem 4.1:- Suppose the assumption $\left(B_{1}\right)-\left(B_{4}\right)$ hold. Then for a given initial measure $q \in C_{H}$, the delay (3.1) admits a solutions $p\left(\bar{S}_{x o}, q\right)$ on $\overline{x_{0} x_{1}}$ for some $x_{1} \in \overline{x_{0} x_{1}}$.

Proof:-Let $\left\{r_{n}\right\},\left(r_{n}>1\right)$ be a decreasing sequence of real numbers such that $r_{n} \rightarrow 1$ as $n \rightarrow \infty$ as

$$
S_{\text {roxo }}>S_{r_{1} x_{0}}>\ldots \ldots S_{r_{n} x_{0}}>\ldots \ldots \ldots
$$

Then we have $\lim _{n \rightarrow \infty} \mu\left(\left\{S r_{n} x_{0}-S x_{0}\right\}\right)=0$

There fore, these exists a real number $\mathrm{r}$ and a point $x_{1}=r x_{0}$ such that

$$
\int w(x) d \mu<H-\|q\|-\left\|h_{k}\right\| L_{\mu}^{1} m_{1}
$$

Where $m_{1}=\mu\left(\overline{x_{0} x_{1}}\right)$. This is possible by virtue of $\left(A_{1}\right)$ and the positiveness of $\mu$.

How in the Banach space $A C^{1}\left(S x_{1}, M n_{1}\right)$ we define a subset $\mathrm{S}$ by

$$
S=\left\{p \in A C^{1}\left(S_{z}, M_{z}\right) \mid p(E)=q(E)\right.
$$

if $E \in M_{0}$ and $\left.\|p\| \leq k\right\}$

Where, the constant $\mathrm{k}$ is given by

$$
k=\|q\|+\left\|h_{k}\right\|_{L_{\mu}^{1}} m_{1}
$$

From (4.2) and (4.3) it follows that $\|p\|<H$ for all $p \in S$. Define an operator $\mathrm{T}$ from $\mathrm{S}$ into $A C^{1}\left(S x_{1}, M x_{1}\right)$ by

$$
T p(E)=\left\{\begin{array}{c}
\int\left(\int_{S_{x_{\omega}}} f\left(t, p\left(\bar{S}_{t}\right), \int_{S_{t_{\omega}}} k\left(t, p\left(\bar{S}_{t_{\omega}}\right)\right) d \mu\right) d \mu, \text { if } E \in M_{z}, E \subset \overline{x_{0} z}\right. \\
q(E), \quad \text { if } E \in M_{0}
\end{array}\right.
$$

We shall show that the operator $\mathrm{T}$ satisfies all the conditions of theorem (3.1) on S.

Step I:- We show that + continuously maps $S$ into itself. First show that the operator $\mathrm{T}$ maps $\mathrm{S}$ into itself. Let $\mathrm{p} t \mathrm{~S}$ be arbitrary and let $E \in M x_{1}$. Then there are sets $F \in M_{0}$ and $G \in M x_{1}, \quad G \subset \overline{x_{0} x_{1}}$ such that $E=F U G$.

Then 


$$
|T p(E)|_{n} \leq|q(F)|_{n}+\iint_{G}\left(\int_{\bar{S}_{x_{\omega}}} f\left(t, p\left(\bar{S}_{t}\right), \int_{\bar{S}_{t_{\omega}}} k\left(t, p\left(\bar{S}_{t_{\omega}}\right)\right) d \mu\right) d \mu \leq\|q\|+\iint_{E}\left(\int_{\bar{S}_{x_{\omega}}} h_{k}(t) d \mu\right) d \mu \leq\|q\|+\int_{\bar{x}_{0} x_{1}}\|h\|_{L_{\mu}^{1}} d \mu \leq\|q\|+\left\|h_{k}\right\|_{L_{\mu}^{1}} m_{1}\right.
$$

for all $E \in M x_{1}$. From the definition of the norm in $A C^{1}\left(S x_{1}, M x_{1}\right)$, one has $\|T p\| \leq\|q\|+\left\|h_{k}\right\|_{L_{\mu}^{1}} m_{1}=K$.

This show that $\mathrm{T}$ maps $\mathrm{S}$ into itself. Now we show that $\mathrm{T}$ is continuous on S. Let $\left\{p_{n}\right\}$ be a sequence of vector measures in $\mathrm{S}$ converging to vector measure $\mathrm{p}$, that is, $\lim _{n \rightarrow \infty}\left\|p_{n}-p\right\|=0$. Then for $\eta>0$, by hypothesis $\left(A_{4}\right)$, these exists a $\delta>0$ such that

$$
\left\|p_{n}-p\right\|<\delta \Rightarrow \mid f\left(x, p_{n}\left(\bar{S}_{x}\right), \int_{\bar{S}_{x_{\omega}}} k\left(t, p_{n}\left(\bar{S}_{t}\right)\right) d \mu-f\left(x, p\left(\bar{S}_{x}\right), \int_{\bar{S}_{x_{\omega}}} k\left(t, p\left(\bar{S}_{t}\right)\right) d \mu \mid<\frac{\eta}{m_{1}^{2}}\right.\right.
$$

Therefore, fore any $E \in M x_{1}$,

$$
\begin{aligned}
& \left|T p_{n}(E)-T p(E)\right|_{n} \leq \int_{E}\left(\int_{S_{x_{\omega}}} \mid t\left(t, p_{n}\left(\bar{S}_{x}\right), \int_{S_{t_{\omega}}} k\left(t, p_{n}\left(\bar{S}_{t}\right)_{\omega}\right) \mid d \mu-F\left(t, p\left(\bar{S}_{x}\right), \int_{\bar{S}_{t_{\omega}}} k\left(t, p\left(\bar{S}_{t_{\omega}}\right)\right) d \mu \mid d \mu\right) d \mu\right.\right. \\
& \leq \int_{E}\left(\int_{S_{x_{\omega}}} \frac{\eta}{m_{1}^{2}} d \mu\right) d \mu<\eta
\end{aligned}
$$

$\left\|p_{n}-p\right\|<\delta$. This shows that $\mathrm{T}$ is a continuous operator on $\mathrm{S}$.

Step II:- Next, we show that $\mathrm{T}(\mathrm{S})$ is a uniformly bounded

and set in $A C^{1}\left(S x_{1}, M x_{1}\right)$. Now as in step $\mathrm{I}$, it is proved that $\mathrm{T}$ (S) is a subset of $\mathrm{S}$ and hence it is uniformly bounded set in $A C^{1}\left(S x_{1}, M x_{1}\right)$. Now by the definition of the map $\mathrm{T}$ are has

$$
T p(E)=\left\{\begin{array}{l}
\iint_{S_{x_{\omega}}}\left(\int_{E} f\left(t, p\left(\bar{S}_{t}\right), \int_{S_{t_{\omega}}} k\left(t, p\left(\bar{S}_{t_{\omega}}\right)\right) d \mu\right) d \mu, \text { if } E \in M_{z}, E \subset \overline{x_{0} x_{1}}\right. \\
q(E) \quad, \quad \text { if } E \in M_{0}
\end{array}\right.
$$

Further we show that $\mathrm{T}(\mathrm{S})$ is an equi - continuous set in $A c^{1}\left(S x_{1}, M x_{1}\right)$

Let $E_{1}, E_{2} \in M_{z}$ then there are sets $F_{1}, F_{2} \in M_{0}$ and $G_{1}, G_{2} \in M x_{1}$ with $G_{1}, G_{2} \subset \overline{x_{0} x_{1}}$, and

$$
\text { FinGi= } \phi, \quad i=1,2 \text {. }
$$

We know that the set - identities

$$
\begin{aligned}
& G_{1}=\left(G_{1}-G_{2}\right) \cup\left(G_{2} \cap G_{1}\right) \\
& G_{2}=\left(G_{2}-G_{1}\right) \cup\left(G_{2} \cap G_{1}\right)
\end{aligned}
$$

Therefore, we have

$$
\begin{aligned}
& T p\left(E_{1}\right)-T p\left(E_{2}\right)=q\left(F_{1}\right)-q\left(F_{2}\right) \\
& \quad+\int_{G_{1}-G_{2}}\left(\int_{\bar{S}_{x_{\omega}}} t\left(t, p\left(S_{t}\right), \int_{\bar{S}_{t_{\omega}}} k\left(t, p\left(\bar{S}_{t_{\omega}}\right)\right) d \mu\right) d \mu\right. \\
& \quad-\int_{G_{2}-G_{1}}\left(\int_{\bar{S}_{\bar{S}_{\omega}}} f\left(t, p\left(S_{t}\right), \int_{\bar{S}_{t_{\omega}}} k\left(t, p\left(\bar{S}_{t_{\omega}}\right)\right) d \mu\right) d \mu\right.
\end{aligned}
$$

Since $f(x, y, z)$ is $L_{\mu}^{1}$ - Caratheodory, we have that

$$
\begin{aligned}
& \left|\operatorname{Tp}\left(E_{1}\right)-T p\left(E_{2}\right)\right|_{n} \leq\left|q\left(F_{1}\right)-q\left(F_{2}\right)\right|_{n}+ \\
& \int_{G_{1} \Delta G_{2}}\left(\int_{\bar{S}_{x_{\omega}}} \mid f\left(x, p\left(\bar{S}_{x}\right), \int_{\bar{S}_{x_{\omega}}} k\left(x,\left.p_{n}\left(\bar{S}_{x_{\omega}}\right)\right|_{n} d \mu\right) d \mu\right.\right. \\
& \leq\left|q\left(F_{1}\right)-q\left(F_{2}\right)\right|+\int_{G_{1} \Delta G_{2}}\left(\int_{\bar{S}_{x_{\omega}}} h_{r}(x) d \mu\right) d \mu \\
& \leq\left|q\left(F_{1}\right)-q\left(F_{2}\right)\right|+\int_{G_{1} \Delta G_{2}}\left\|h_{r}\right\|_{L_{\mu}^{1}} d \mu
\end{aligned}
$$

Assume that $d\left(E_{1}, E_{2}\right)=|\mu|_{n}\left(E_{1} \Delta E_{2}\right) \rightarrow 0$.

Then we have $E_{1} \rightarrow E_{2}$ and consequently $F_{1} \rightarrow F_{2}$ and $|\mu|_{n}\left(G_{1} \Delta G_{2}\right) \rightarrow 0$. From the continuity of on $M_{0}$ it follows that

$$
\begin{aligned}
\left|T p\left(E_{1}\right)-T p\left(E_{2}\right)\right|_{n} \leq\left|q\left(F_{1}\right)-q\left(F_{2}\right)\right|_{n}+ \\
\int_{G_{1} \Delta G_{2}}\left\|h_{r}\right\|_{L_{\mu}^{\prime}} d \mu \rightarrow 0 \quad \text { as } E_{1} \rightarrow E_{2}
\end{aligned}
$$

This show that $\mathrm{T}(\mathrm{S})$ is an equi-continuous set on $A C^{1}\left(S_{z}, M_{z}\right)$, thus $\mathrm{T}$ (s) is uniformly bounded and equicontinuous set in $A C^{\prime}\left(S_{z}, M_{z}\right)$ so it is compact in the norm topology on $A C^{1}\left(S x_{1}, M x_{1}\right)$. Now an application of Arzela 
Ascoli Theorem yields that $\mathrm{T}(\mathrm{S})$ is a compact subset of $A C^{1}\left(S x_{1}, M x_{1}\right)$. As a result, $\mathrm{T}$ is a continuous and compact operator on essences, an application of theorem 3.1 yields that the operator equation $p=T p$ has a solution is $\mathrm{S}$. As a result, the delay (3.1) has a solution on $\overline{x_{0} x_{1}}$. This completes the proof.

\section{Extension of Stability}

A solution of the delay (3.1) so obtained can be extended to the larger segment, whenever $\mu\left(\left\{x_{1}\right\}\right)=0$. An existence result in this condition is.

Theorem 5.1. Under the hypothesis of theorem (4.1) let $p\left(\bar{S} x_{0}, q\right)$ be solution of the delay (4.3.1) on $\overline{x_{0} x_{1}}$. Then the larger segment if $\mu\left(\left\{x_{1}\right\}\right)=0$

Proof :-

Definition 5.1:-

We consider the following assumptions.

$\left(H_{1}\right)$ The function $f: S_{z} \times B_{x} \rightarrow R$ is $\mu$-integrable and $f(x, 0)=0$ for all $x \in S_{z}$ for some $z>x_{0}$.

$\left(H_{2}\right)$ Given $\delta>0$, there exists $a \in>0$ such that

$$
\left|f(x, y, z)-f\left(x, y_{z}, z_{z}\right)\right| \leq \delta\left[\left|y_{2}-z_{1}\right|+\left|y_{2}-z_{2}\right|\right]
$$

For all $y_{1}, y_{2}, z_{1}, z_{2} \in R$ with

$$
\begin{aligned}
\left|y_{1}\right| \subseteq \mathcal{E} \quad, \quad\left|y_{2}\right| \leq \varepsilon,\left|z_{1}\right| \leq \varepsilon,\left|z_{2}\right| \leq \varepsilon \\
T p(E)=\left\{\begin{array}{l}
\int\left(\int_{S_{x_{\omega}}} t\left(t, p\left(\bar{S}_{t}\right), \int_{S_{t_{\omega}}} k\left(t, p\left(\bar{S}_{t_{\omega}}\right) d \mu\right) d \mu\right) d \mu \text { if } E \in M_{z}, E \subset \overline{x_{0} z}\right. \\
q(E) ; \text { if } E \in M_{0}
\end{array}\right.
\end{aligned}
$$

Now if $E \in M_{z}$, then there are two disjoint sets $\mathrm{F}$ and $\mathrm{G}$ in $M_{z}$ such that

$$
E=F \cup G, F \in M_{0}, G \subset \overline{x_{0} z}
$$

Hence for $E \in M_{z}$, from (5.2) and (5.3) it follows that

$$
|T p(E)| \leq|q|(E)+\int_{G}\left(\int_{\bar{S}_{x_{\omega}}} \mid f\left(t, p\left(\bar{S}_{t}\right), \int k\left(t, p\left(\bar{S}_{t_{\omega}}\right) d \mu\right) \mid d \mu\right) d \mu \leq\|q\|+2 \iint_{G}\left(\int_{\bar{S}_{x_{\omega}}} \delta \in d \mu\right) d \mu \leq\|q\|+2 m^{2} \delta \varepsilon \leq b \varepsilon+(1-b) \varepsilon=\varepsilon\right.
$$

For all $p \in \bar{S}(\varepsilon)$, where $\|q\| \leq b \varepsilon$ and $\delta=\frac{1-b}{2 m^{2}}$

Therefore $\|T p\| \leq \varepsilon$ for all $p \in \bar{S}(\varepsilon)$. This shows that T maps $\mathrm{S}$ (e) into itself. Next, we show that $\mathrm{T}$ is a contraction operator on $\bar{S}(\varepsilon)$. Let $p_{1}, p_{2} \in \bar{S}(\varepsilon)$. Then by $\left(H_{2}\right)$,

$$
\left|T p_{1}(E)-T p_{2}(E)\right| \leq\left|\iint_{E}\left(\int_{\bar{S}_{x_{\omega}}}\left(t, p_{1}\left(\bar{S}_{t}\right), \int k\left(t, p\left(\bar{S}_{t_{\omega}}\right)\right) d \mu\right) d \mu\right) d \mu-\int_{E}\left(\int_{S_{x_{\omega}}} f\left(t, p_{2}\left(\bar{S}_{t}\right), \int k\left(t, p_{2}\left(\bar{S}_{t_{\omega}}\right)\right) d \mu\right) d \mu\right) d \mu\right|
$$




$$
\begin{gathered}
\leq \int_{E}\left(\int_{S_{x_{\omega}}} \mid f\left(t, p_{1}\left(\bar{S}_{t}\right), \int k\left(t, p_{1}\left(\bar{S}_{t_{\omega}}\right)\right)-f\left(t, p_{2}\left(\bar{S}_{t}\right), \int k\left(t, p_{2}\left(\bar{S}_{t_{\omega}}\right)\right) d \mu\right) \mid d \mu\right) d \mu\right. \\
\leq \int_{E}\left(\int_{S_{x_{\omega}}} \delta\left[\left|p_{1}\left(\bar{S}_{t}\right)-p_{2}\left(\bar{S}_{t}\right)\right|+\left|\int k\left(t, p_{1}\left(\bar{S}_{t_{\omega}}\right)\right) d \mu-\int k\left(t, p_{2}\left(\bar{S}_{\omega}\right)\right) d \mu\right|\right] d \mu\right) d \mu \leq 2 \int_{E}\left(\int_{S_{x_{\omega}}} \delta\left\|p_{1}-p_{2}\right\| d \mu\right) d \mu \\
\leq 2 m^{2} \delta\left\|p_{1}-p_{2}\right\| \leq(1-b)\left\|p_{1}-p_{2}\right\|
\end{gathered}
$$

For all $E \in M_{z}, E \subset \overline{x_{0} z}$. This further implies that.

$$
\left\|T p_{1}-T p_{2}\right\| \leq \alpha\left\|p_{1}-p_{2}\right\|
$$

Where, $\alpha=(1-b)<1$. This shows that $\mathrm{T}$ is a contraction operator on $\bar{S}(\varepsilon)$ with the contraction constants $\alpha$. Therefore, by an application of contraction mapping principle, there is a unique solution $p\left(\bar{S} x_{(0)} q\right)$ of the delay (3.1) satisfying $\|p\| \leq \mathcal{E}$ whenever $\|q\| \leq b \mathcal{E}$. This completes the proof.

Example 5.1:- Let $X=R \mu$ the Lebesgue measure on $R, \bar{S}_{x}=[0, x] x>0$ and $q(E)=\mu(E), E \subset[0,2]$. Consider the delay AMIGDE

$$
\frac{d p}{d \mu}=6 \int_{\bar{S}_{x-\frac{1}{2}}} p\left(\bar{S}_{t-1}\right) d \mu
$$

and

$$
p(E)=q(E), E \subset\left[0, \frac{1}{2}\right]
$$

Here $\omega=\frac{1}{2}$ for $0 \leq x \leq 2$, we observe that

$$
p\left(\bar{S}_{x}\right)=p(0, x)=q([0, x])=x
$$

If $x \in[1,2]$ then we have

$$
\begin{gathered}
p\left(\bar{S}_{x}\right)=q\left(\bar{S}_{1}\right)+\int_{[1, x]}\left(\int_{\left[x-t-\frac{1}{2}\right]} 6 p\left(\bar{S}_{s-\frac{1}{2}}\right) d s\right) d t=1+6 \int_{1}^{x}\left(\int_{1}^{t-\frac{1}{2}}\left(s-\frac{1}{2}\right) d s\right) d t \\
=1+\frac{6}{2} \int_{1}^{x}\left[(t-1)^{2}-\frac{1}{4}\right] d t=1+3\left[\left(\frac{t-1}{3}\right)^{3}-\frac{1}{4} t\right]_{1}^{x}=(x-1)^{3}-\frac{3}{4} x+\frac{7}{4}
\end{gathered}
$$

In this way the solution $\mathrm{p}$ for the linear delay AMIGDE (5.6) can be found recursively on $[0, \infty]$.

\section{Acknowledgments}

The authors are thankful to the referee for their valuable suggestions. The author S. S. Bellale is also thankful to UGC, New Delhi (MRP-Major, R.N.41-750, 2012) for their financial support.

\section{References}

[1] B. C. Dhage "On abstract measure integro-differential equations”, J. Math. Phy. Sci.20 (1986), 367 - 380

[2] B. C. Dhage "On system of abstract measure integro differential inequations and applications", Bull. Inst. Math. Acad. Sinica18 (1989), 65 - 75

[3] B. C. Dhage "Mixed monotonicity theorems for a system of abstract of measure delay integro - differential equations", An. Stint. Univ. “Al. I. Cuza” IasiXLII (1996) 355 - 366

[4] B. C. Dhage and S. S. Bellale, "Abstract measure integro differential equations", Global Jour Math. Anal.1 (2007), 91 108 .
[5] B. C. Dhage and S. S. Bellale, "Existence theorem for perturbd abstract measure differential equations", Nonlinear Analysis, 71(2009),e319-e328

[6] S. S. Bellale, "Hybrid Fixed Point Theorem For Abstract Measure Differential Equation”, World Academy Of Science, Engineering and Technology, 73(2013) 782-785 ISSNe2010-3778

[7] B. C. Dhage, D. N. Chate and S. K. Ntouyas, "Abstract measure differential equations", Dynamic Systems \& Appl. 13 (2004) $105-108$.

[8] J. Dagudji and A. Granas, "Fixed point Theory", Monograhie Math. PNW. Warsaw 1982.

[9] S. R. Joshi, "A system of abstract measure delay differential equations", J. Math. Phy. Sci. 13 (1979), 496 - 506.

[10] S. R. Joshi and S. G. Deo, "On abstract measure delay differential equations", An. Stint. Univ. Al I. CuzaIassiXXVI (1980), $327-335$.

[11] W. Rudin, "Real and Complex, Analysis, McGraw", - Hill Inc.New York, 1966.

[12] R. R. Sharma, "An abstract measure differential equation", Proc, Amer, Math. Soc. 32 (1972) 503 - 510.

[13] R. R. Sharma, "A measure differential inequality with applications", Proc. Amer. Math. Soc. 48 (1975) 87 - 97. 
[14] G. R. Shendge and S. R. Joshi, "Abstract measure differential inequalities and applications", Acta Math Hung. 41 (1983), 53 -54 .
[15] D. R. Smart, "Fixed point Theorems", Cambridge Unive. Press, Cambridge 1974. 